\title{
The Clinical Dilemma of Incidental Findings on the Low-Resolution CT Images from SPECT/CT MPI Studies
}

\author{
Joanne Coward, Julie Nightingale, and Peter Hogg \\ Directorate of Radiography, School of Health Sciences, University of Salford, Salford, United Kingdom
}

\begin{abstract}
CE credit: For CE credit, you can access the test for this article, as well as additional JNMT CE tests, online at https://www.snmmilearningcenter.org. Complete the test online no later than September 2019. Your online test will be scored immediately. You may make 3 attempts to pass the test and must answer $80 \%$ of the questions correctly to receive $1.0 \mathrm{CEH}$ (Continuing Education Hour) credit. SNMMI members will have their CEH credit added to their VOICE transcript automatically; nonmembers will be able to print out a CE certificate upon successfully completing the test. The online test is free to SNMMI members; nonmembers must pay $\$ 15.00$ by credit card when logging onto the website to take the test.
\end{abstract}

Incidental findings are common in medical imaging. There is a particularly high prevalence of incidental findings within the thorax, the most frequent being pulmonary nodules. Although pulmonary nodules have the potential to be malignant, most are benign, resulting in a high number of false-positive findings. Low-resolution CT images produced for attenuation correction of SPECT images are essentially a by-product of the imaging process. The high number of false-positive incidental findings detected on these attenuation-correction images causes a reporting dilemma. Early detection of cancer can be beneficial, but falsepositive findings and overdiagnosis can be detrimental to the patient. Attenuation-correction CT images are not of diagnostic quality, and further diagnostic tests are usually necessary for a definitive diagnosis to be reached. Given the high number of falsepositive findings, the psychologic effect on the patient should be considered. This review recommends caution when the findings on attenuation-correction CT images are routinely reported.

Key Words: SPECT; low-resolution CT; myocardial perfusion imaging

J Nucl Med Technol 2016; 44:167-172

DOI: 10.2967/jnmt.116.174557

M edical imaging uses a range of modalities that yield both anatomic and functional information (1). Visual correlation of images acquired using separate modalities can provide more information than images from a single modality alone, though there is an inevitable risk of misregistration between images that have been acquired during different imaging sessions. The development of hybrid imaging has led to the integration of two modalities in one machine, allowing coregistration of images that have been

\footnotetext{
Received Feb. 23, 2016; revision accepted Apr. 4, 2016.

For correspondence or reprints contact: Joanne Coward, Directorate of Radiography, School of Health Sciences, Allerton Building, University of Salford, Salford M6 6PU, United Kingdom.

E-mail: j.coward@salford.ac.uk

Published online Apr. 21, 2016.

COPYRIGHT (C) 2016 by the Society of Nuclear Medicine and Molecular Imaging, Inc.
}

acquired in a single session $(1,2)$. This allows direct correlation of anatomic and functional information, increasing sensitivity and specificity while adding clarity to indeterminate cases (3). SPECT/CT offers an excellent example of hybrid imaging, combining the functional ability of SPECT with the anatomic ability of CT.

The strength of SPECT lies in gaining pathophysiologic detail in a minimally invasive way (4). Pathologic processes can be identified by uptake of radiopharmaceuticals, but the precise location is often difficult to ascertain because of low image resolution and a lack of anatomic landmarks. Moreover, uptake on some scans is often nonspecific, revealing abnormalities but not their specific cause. CT can provide a useful means of localization, also enabling additional characterization by virtue of providing a site-specific correspondence between anatomic and physiologic information.

SPECT images are susceptible to artifacts as a result of attenuation deficits due to scatter and absorption of photons (5-7). To improve image quantification and reduce attenuation artifacts, a low-dose CT acquisition can be performed and then used to correct the SPECT images for attenuation. $\mathrm{CT}$ attenuation correction is now in common use because it often improves image quality and increases overall diagnostic accuracy (5).

There are therefore 3 distinct reasons why CT may be combined with SPECT: First, CT can be used to characterize an abnormality seen on the SPECT images. This use usually requires a diagnostic-quality (high-resolution) CT scan. Second, CT can be used to localize an abnormality seen on the SPECT images. Third, CT can be used to correct the SPECT images for attenuation. This use requires only a lowresolution CT scan, typically using a much lower ionizing radiation dose than that required for diagnostic-quality CT. Although the images are considered to be nondiagnostic, they often reveal incidental findings.

In this article we discuss incidental findings on the lowresolution CT images produced for attenuation correction of SPECT images, with particular emphasis on myocardial perfusion imaging (MPI). 


\section{DEFINITIONS AND FREQUENCY OF INCIDENTAL FINDINGS}

An incidental finding can be defined as an unsuspected finding that is not related to the clinical reason for performing the diagnostic test $(8,9)$. Examples of incidental findings that can be identified on attenuation-correction CT images of the chest include coronary vessel calcification, vascular anomalies, valve replacement, pacemakers, atelectasis, effusion, consolidation, lobar collapse, nodules, masses, pulmonary metastases, ground glass opacities, and aortic aneurysm (20).

Incidental findings may or may not already be known from previous diagnostic tests. Findings that are already known to the clinician are likely to have a management strategy in place and therefore often do not require further investigation. Previously unknown incidental findings are termed new incidental findings and are likely to fall into 1 of 3 categories: clinically significant, clinically insignificant, or indeterminate. Clinically significant incidental findings are highly suspected of having an underlying pathology that may negatively affect patient wellbeing, requiring further investigation (9).

The frequency of incidental findings on attenuationcorrection CT images is particularly high within the thorax (10), possibly because of the inherent contrast resolution and low attenuation of the x-ray beam through this area. Lung cancer shares comorbidities with heart disease-the clinical reason that these patients are being scanned. It is therefore not surprising that extracardiac pathology is frequently detected.

The high number of incidental findings identified on attenuation-correction CT images causes a dilemma. The acquisition is intended for attenuation correction alone and not for evaluation, and the relatively low resolution of the images means that characterization of findings is often not possible. Additionally, unlike diagnostic-quality CT images of the chest and abdomen, attenuation-correction images are likely to have breathing artifacts because patients are not required to hold their breath. The craniocaudal range of the acquisition is also limited to the cardiac area only; detection of incidental findings is therefore also restricted.

\section{INCIDENTAL FINDINGS ON SPECT/CT MPI}

The number of incidental findings on the attenuationcorrection CT images produced during SPECT/CT MPI is noteworthy, and the percentage of clinically significant and indeterminate findings can often be as high as 10\% (10).

A high proportion of findings are pulmonary nodules (11). Although most lung nodules are benign, they have the potential to develop into lung cancer (12). Attenuationcorrection CT images, which often reveal lung nodules as well as other pathologies, were never intended for radiologic reporting but there is growing evidence to suggest that they should be (10).

Lung cancer is the leading cause of death in most countries, including the United States $(12,13)$. Early lung cancer is often asymptomatic, with symptoms usually arising only when the disease is at an advanced stage. The prognosis for lung cancer is consequently poor (13). However, the prognosis for nonsmall cell lung cancer improves significantly if it is detected at an early stage when surgical resection is possible $(12,13)$. This is not the case with small cell lung cancer. In the management of pulmonary nodules, the recommendations that have been developed from lung cancer screening trials suggest a necessary balance between early intervention to reduce mortality from lung cancer and the risk of early morbidity and mortality from intervention due to false-positive findings and overdiagnosis (11).

The high prevalence of lung nodules on CT of the chest is of particular concern during SPECT/CT MPI because any such findings will be incidental and may signpost a patient to an alternative or additional-care pathway. Although there is an argument for early treatment of lung cancer, the detection of lung nodules on attenuation-correction CT images does not necessarily result in reduced mortality from lung cancer. A high proportion of lung nodules are ultimately found to be benign, and the patient may not benefit, and may actually be harmed, from follow-up of these nodules.

This sentiment is echoed by the outcomes of lung cancer screening trials; for a screening program to be effective, mortality must be reduced and the benefits to the patient must outweigh the risks (12). The National Lung Screening Trial has demonstrated that it is possible to reduce mortality from lung cancer using low-dose CT screening in place of chest radiography and sputum tests (14). However, in doing so, there is a high rate of false-positive findings and overdiagnosis, as well as an increased radiation burden to the patient (15). Overdiagnosis of indolent tumors that would not have become symptomatic during the patient's lifetime have cost implications as well as raising patient anxiety and morbidity in the same way that false-positive findings might (16). Consequently, several organizations have decided not to implement screening programs. Where screening programs have been implemented, they are focused specifically on the population at high risk (17).

These considerations add to the dilemma of whether clinicians should review the attenuation-correction images. Reviewing them and providing a report may possibly enable early diagnosis of pathology and potentially a better prognosis. However, identifying incidental findings may increase risk to the patient without necessarily providing any benefit. Further diagnostic examinations are often associated with an increase in ionizing radiation dose and possibly invasive procedures that may carry physical risk and cause psychologic harm to the patient $(9,18,19)$. Furthermore, only a small percentage of these incidental findings are significant at definitive diagnosis $(9,20,21)$.

\section{CONTRAST ISSUES}

There is often poor inherent contrast resolution between organs and soft tissues within the body due to relatively small differences in density. The use of contrast agents artificially improves contrast resolution between soft-tissue 
structures. The iodination of the contrast agent increases the density within the organs and so will affect the extent to which the x-ray beam is attenuated. Different organs take up contrast at different rates, resulting in an increase in contrast resolution on the CT images. Similarly, pathologic tissue can demonstrate selective uptake of contrast agents; tumors that are highly vascular tend to enhance avidly whereas ischemic tissue tends not to enhance. This selective uptake improves demonstration of pathology in relation to normal tissue and aids characterization of the pathology. For this reason, it is unusual to perform diagnostic CT of the chest, abdomen, and pelvis without the use of an intravenous contrast agent.

The use of intravenous contrast agents with SPECT/CT is not without problems. Contrast agents will attenuate $\gamma$-rays in the same way that they attenuate $\mathrm{x}$-rays. The result is degradation of the quality of the SPECT images. Contraindications relating to the use of iodinated intravenous contrast agents also exist.

It is because of the acquisition parameters used, along with the lack of an intravenous contrast agent to enhance contrast resolution, that attenuation-correction CT images are not considered diagnostic.

\section{IMAGE-QUALITY ISSUES}

The quality of attenuation-correction CT images is directly related to how the images have been acquired, typically with a low tube current $(\mathrm{mA})$ and a wide slice. The resulting lowresolution $\mathrm{CT}$ images enable attenuation correction to be performed. The long acquisition time results in motion artifacts from breathing. The nature of incidental findings can be difficult to determine, resulting in a high number of false-positive findings.

As technology has progressed, multidetector CT scanners have been utilized in some hybrid systems. Multidetector CT offers increased technologic capabilities with the potential to produce images superior in quality to the attenuation-correction images typically associated with earlier systems. The potential to acquire narrow slices, reduce imaging time, and increase the tube current has provided the option of improving image quality to a level that, in some cases, is comparable to that of diagnostic CT. Having this option has inevitably led to differences in the quality of the attenuation-correction images acquired in different departments, related not only to the capability of the scanner but also to the way the parameters have been optimized (20). Ethical considerations arise when CT image quality is improved in this way, because there will be an inevitable increase in ionizing radiation dose to the patient in exchange for an uncertain gain.

\section{Radiation-Dose Considerations}

CT image quality is dependent on how the data are acquired, reconstructed, and viewed. Ideally, we would choose the best image quality possible, but this would involve changing the acquisition parameters in a way that would greatly increase the radiation dose to the patient. Therefore, it is necessary to use a technique called optimization (22), the aim of which is to produce the required image quality with the lowest possible radiation dose. The result is not necessarily the best image quality or the lowest radiation dose but a compromise producing images tailored to the purpose for which they are acquired.

Diagnostic CT images are of sufficient quality for a diagnosis to be made. It is usually necessary for these images to have good spatial and contrast resolution and to be free of artifacts. This requirement does not apply to the attenuationcorrection CT images acquired for MPI. Because the acquisition is merely for attenuation-correction purposes, the image quality can be significantly lower.

As shown in Table 1, the tube current used for attenuationcorrection CT is much lower than that for diagnostic CT, enabling the radiation dose to the patient to be reduced. The resultant CT images will be noisy and have poor contrast resolution. The slices are usually considerably wider for attenuation-correction CT than for diagnostic CT. This greater width also has an effect on image quality, reducing the spatial resolution (ability to determine fine detail) but improving the contrast resolution. The long rotation time frequently associated with some of the older SPECT/CT systems results in a longer overall scanning time, rendering the attenuationcorrection images susceptible to motion artifacts, especially within the thorax and abdomen. Although the presence of motion artifacts has no significance in CT images acquired for attenuation-correction purposes, it does become relevant when the intent is to make a diagnosis from the images.

\section{Diagnostic-Value Considerations}

Studies investigating the influence of differences in attenuation-correction CT image quality on detection of

TABLE 1

Differences in Acquisition Parameters Between Diagnostic CT and Attenuation-Correction CT for SPECT/CT MPI

\begin{tabular}{lcr}
\hline \multicolumn{1}{c}{ Parameter } & Diagnostic CT & Attenuation-correction CT \\
\hline Voltage $(\mathrm{kV})$ & 120 & 120 \\
Tube current $(\mathrm{mA})$ & Automated $(\sim 300-400)$ & $1.5-33$ \\
Rotation time $(\mathrm{s})$ & 0.33 & $1.5-30$ \\
Effective mAs & $100-130$ (dependent on automated $\mathrm{mA})$ & $24-50$ \\
Slice thickness $(\mathrm{mm})$ & & \\
Acquired & $0.5-1$ & $5-10$ \\
Reconstructed & 3 & $5-10$ \\
Pitch & $0.75-1$ & $1-2$ \\
\hline
\end{tabular}


nodules in chest phantoms have been performed using the Free-Response Receiver Operating Characteristic method. This method assesses observer performance and captures observers' ability to decide where the lesion is and to rate their confidence in this decision.

In a lung phantom study, varying the tube current $(1,1.5$, 2 , and $2.5 \mathrm{~mA}$ ) of a SPECT/CT system while keeping all the other parameters unchanged had no statistically significant effect on lesion-detection performance (23). In a further lung phantom study involving a range of SPECT/CT systems, there was a difference in lesion detectability relative to the capability of the CT unit (24). These results were reproduced in a study using the same chest phantom on two different SPECT/CT systems. Here, lesion detection was more reliable on one system than on the other and was found to be related to the reconstruction algorithms used on one of the CT units rather than the acquisition parameters (25). The phantom was stationary in all studies and so did not truly represent the clinical situation in which motion artifacts from breathing would degrade the attenuation-correction CT images.

A 2-y multicenter study that took place in 4 nuclear medicine departments in the United Kingdom was granted local approval from each participating hospital and ethical approval from the University of Salford, following advice from the Health Research Authority (26). Positive findings were identified on the attenuation-correction CT images of $962(28 \%)$ of 3,485 patients undergoing SPECT/CT MPI. Of these findings, 824 (24\%) were new. Eighty-four patients $(2.4 \%)$ had findings that were thought to be clinically significant at the time of the imaging and had not been discovered previously, but only 10 patients $(0.29 \%)$ had findings that were confirmed to be clinically significant at definitive diagnosis. In this study, 74 of 84 patients had false-positive findings that involved follow-up diagnostic tests and possibly intervention before a definitive negative outcome was reached. The implied increased physical and psychologic risk to the patient raises the question of whether the attenuation-correction CT findings should be reported. The positive predictive value across all the centers was found to be low, and one center consequently stopped reporting the attenuation-correction CT findings. Statistically, the positive predictive values did not significantly differ between attenuation-correction CT images acquired on a lower-resolution machine and those acquired on a higher-resolution machine. The study concluded that routine reporting of attenuation-correction CT findings was not beneficial. The information from this study appears to be unique; no other similar clinical studies were identified.

Whether to report attenuation-correction CT findings is a common dilemma because the detection of early pathology can lead to an improved prognosis. However, further investigations to characterize the findings can be costly and can increase the radiation burden and other risks to the patient (27).

\section{PSYCHOLOGIC EFFECTS}

False-positive incidental findings or findings that result in overdiagnosis can increase patient anxiety. Although diagnosis of malignancies at an early stage can potentially reduce patient mortality, only a very small percentage of findings on attenuation-correction CT images have been found to be malignant or detrimental to the patient at definitive diagnosis (28).

There is only limited literature surrounding the psychologic effects on patients who learn of incidental SPECT/CT findings. There is, however, an abundance of literature relating to the psychologic effect of a recent diagnosis of cancer, or the wait for a definitive diagnosis, and some parallels can be drawn to patients with incidental findings.

Patients who have incidental findings on a screening examination or are being imaged because of suspected cancer will usually have a care pathway and support structure in place, typically involving nursing, medical, and other professionals who are available to help patients and their families. This is often not the case for patients who have incidental findings on SPECT/CT. Such patients will receive limited support because of the unexpected nature of the findings.

Pulmonary nodules are one of the most frequent incidental findings on SPECT/CT of the thorax $(19,29)$. These nodules can be single or multiple and can be of unknown significance. Most are benign, but if indeterminate the patient will require surveillance that may continue for $2-3$ y (30). Almost all patients who are told they have a pulmonary nodule assume they have cancer (16). Just raising the possibility of cancer can be threatening (17) and can lead to distress until, and possibly beyond, definitive diagnosis (18).

Pulmonary nodules are of particular clinical relevance (8) because they do have the potential to become malignant, although most will be benign (31). Further management of pulmonary nodules is based on their size, with the likelihood of malignancy increasing with the size of the nodule $(32,33)$. The indeterminate nature of pulmonary nodules frequently leads to follow-up CT imaging to monitor any change in the size of the nodule. If its size remains stable over a period of $2 \mathrm{y}$, it is considered benign. This lengthy period of repeated imaging inevitably increases the radiation burden to the patient along with the anxiety level. Biopsy of pulmonary nodules, which would give a more definitive answer, is often not possible because of their small size and location within the lung (32). Despite reassurance, the patients may believe they have cancer in the absence of a definitive diagnosis.

Lung cancer screening involves harm as well as benefit, and in some circumstances the harm can outweigh the benefit (33). This also applies to patients with incidental findings on attenuation-correction CT images. The harm from overtreatment of false-positive findings should be kept in mind when considering the benefits of reviewing these images (34). Although patients may gain some reassurance from a negative lung cancer screening, a negative 
attenuation-correction CT scan from SPECT/CT MPI cannot be equally reassuring because of the limited range of chest MPI. A normal attenuation-correction scan does not exclude pathology that falls outside the limited range of the scan. In addition, early detection of lung cancer does not necessarily mean an improved outcome. Approximately $25 \%-30 \%$ of patients will present with potentially curable disease (35), but lung cancers develop quickly and metastasize early, often leading to a poor prognosis (19). Metastases often are present at the time of initial presentation. This frequently poor prognosis also needs to be balanced against the high rate of benign pathology detected on attenuation-correction CT (19).

The psychologic effects on patients who are diagnosed with false-positive findings can discourage patients from attending screening or diagnostic procedures in the future (33). If they have had a significant misdiagnosis, they are likely to lose trust in diagnostic procedures. Psychologic effects can be transient or more persistent. At initial diagnosis the patient is likely to experience anxiety, but over time this tends to develop into depression. Both anxiety and depression can persist before and after treatment (35).

\section{FINANCIAL ISSUES}

The financial impact of routinely reporting the findings of attenuation-correction CT, as well as the time required, needs to be considered. A radiologic report takes time to construct and, in the case of clinically significant findings, is likely to affect future management of the patient. The financial impact is justifiable when there is a net patient benefit, but such does not appear to be the case for attenuationcorrection CT images, with their high yield of false-positive findings. Similarly, the follow-up examinations that may be needed to reach a definitive diagnosis have a cost and require time yet will not necessarily benefit the patient and may, in fact, be a potential cause of harm (36). Thus, the cost-effectiveness of reporting attenuation-correction CT findings is brought into question, along with whether such reporting will benefit or harm the patient.

\section{CONCLUSION}

Attenuation-correction CT yields low-resolution images that are not considered diagnostic. Although they can demonstrate pathology and commonly show incidental findings, the potential for missed or wrongly diagnosed pathology is higher than for diagnostic images.

Incidental findings can be numerous on the attenuationcorrection CT images from SPECT/CT MPI studies and, regardless of the definitive diagnosis, can cause psychologic distress to the patient. One study has called into question the practice of routinely producing a report for these images (28). If the findings are reported, caution should be used and possibly a rider included to point out that the images are lowresolution and not intended for diagnosis. Careful consideration should be given to the potential impact on the patient of a reported abnormal finding.

If the attenuation-correction CT findings are reported, a support structure and appropriate education similar to those in place for screening programs may help patients who have a clinically significant incidental finding.

\section{DISCLOSURE}

No potential conflict of interest relevant to this article was reported.

\section{ACKNOWLEDGMENT}

We thank Prof. Richard Lawson for his time, support, and constructive advice throughout the writing of the manuscript.

\section{REFERENCES}

1. Schillaci O. Hybrid SPECT/CT: a new era for SPECT imaging? Eur J Nucl Med Mol Imaging. 2005;32:521-524.

2. Bhargava P, He G, Samarghandi A, Delpassand ES. Pictorial review of SPECT/ CT imaging in clinical nuclear medicine. Am J Nucl Med Mol Imaging. 2012; 2:221-231.

3. Buck AK, Nekolla S, Ziegler S, et al. SPECT/CT. J Nucl Med. 2008;49:13051319.

4. Erba PA, Israel O. SPECT/CT in infection and inflammation. Clin Transl Imaging. 2014;2:519-535.

5. Pazhenkottil AP, Ghadri JR, Nkoulou RN, et al. Improved outcome prediction by SPECT myocardial perfusion imaging after CT attenuation correction. $\mathrm{J} \mathrm{Nucl}$ Med. 2011;52:196-200.

6. Sil J, Lawson R, Hogg P, Elias M, Kane TP, Taylor R. Reducing dose in CT imaging: theory, practice, and lessons learnt from very low-dose CT. eRADIMAGING website. www.eradimaging.com/site/article.cfm?ID=785. Published August 1, 2012. Accessed May 17, 2016.

7. Ou X, Jiang L, Huang R, Li F, Zhao Z, Li L. Computed tomography attenuation correction improves the risk stratification accuracy of myocardial perfusion imaging. Nucl Med Commun. 2013;34:495-500.

8. Lumbreras B, Donat L, Hernández-Arguado I. Incidental findings in imaging diagnostic tests: a systematic review. Br J Radiol. 2010;83:276-289.

9. Frank L, Quint L. Chest CT incidentalomas: thyroid lesions, enlarged mediastinal lymph nodes and lung nodules. Cancer Imaging. 2012;12:41-48.

10. Goetze S, Pannu HK, Wahl RL. Clinically significant abnormal findings on the low-amperage CT attenuation-corrected myocardial perfusion SPECT-CT studies. J Nucl Med. 2006;47:1312-1318.

11. MacMahon H, Austin JH, Gamsu G, et al. Guidelines for management of small pulmonary nodules detected on CT scans: a statement from the Fleishner Society. Radiology. 2005;237:395-400.

12. Diederich S, Wormanns D, Semik M, et al. Screening for early lung cancer with lowdose spiral CT: prevalence in 817 asymptomatic smokers. Radiology. 2002;222:773-781.

13. Moyer VA. Screening for lung cancer: U.S. preventive services task force recommendation statement. Ann Intern Med. 2014;160:330-338.

14. Aberle DR, Adams AM, Berg CD, et al.; The National Lung Screening Research Team. Reduced lung cancer mortality with low-dose computed tomographic screening. N Engl J Med. 2011;365:395-409.

15. Jemal A, Siegel R, Xu J, Warde E. Cancer statistics. CA Cancer J Clin. 2010; 60:277-300.

16. Patz EF, Pinsky P, Gatsonis C, et al. Overdiagnosis in low-dose computed tomography screening for lung cancer. JAMA Intern Med. 2014;174:269-274.

17. Frauenfelder T, Puhan MA, Lazor R, et al. Early detection of lung cancer: a statement from and expert panel of the Swiss University Hospitals on lung cancer screening. Respiration. 2014;87:254-264.

18. Mold JW, Stein HF. The cascade effect in the clinical care of patients. $N$ Engl J Med. 1986;314:512-514.

19. Budoff MJ, Fischer H, Gopal A. Incidental findings with cardiac CT evaluation: should we read beyond the heart? Catheter Cardiovasc Interv. 2006;68:965-973.

20. Coward J, Lawson R, Kane T, et al. Multi-centre analysis of incidental findings on low-resolution CT attenuation correction images. Br J Radiol. 2014;87:20130701. 
21. White CS. The pros and cons of searching for extracardiac findings at cardiac CT: use of a restricted field of view is acceptable. Radiology. 2011;261:338341.

22. Ionising radiation (medical exposure) regulations 2000 (IRMER). gov.uk website. https://www.gov.uk/government/publications/the-ionising-radiation-medicalexposure-regulations-2000. Published September 5, 2012. Accessed May 17, 2016.

23. Thompson J, Hogg P, Szczepura K, Manning D. Analysis of CT acquisition parameters suitable for use in SPECT/CT: a free response receiver operating characteristic study. Radiography. 2012;18:238-243.

24. Thompson JD, Hogg P, Manning D, Szczepura K, Chakraborty DP. Lesion detection in the CT attenuation correction images of 5 SPECT/CT systems: a multicentre study [abstract]. Eur J Nucl Med Mol Imaging. 2012;39(suppl):S625.

25. Jessop M, Thompson J, Coward J, Sanderud A, Lanca L, Hogg P. Lesion detection performance: comparative analysis of low-dose CT data of the chest on two hybrid imaging systems. J Nucl Med Technol. 2015;43:47-52.

26. Coward J, Lawson R, Kane T, et al. Multicentre analysis of incidental findings on low-resolution $\mathrm{CT}$ attenuation correction images: an extended study. BJR. 2015;88:20150555.

27. Yap KKH, Ramaseshan G, Sutherland T, Eid-Shafik R, Taubman K, Schlicht S. Prevalence of incidental or unexpected findings on low-dose CT performed during routine SPECT/CT nuclear medicine studies. J Med Imaging Radiat Oncol. 2015;59:26-33.
28. Berland LL, Silverman SG, Gore RM, et al. Managing incidental findings on abdominal CT: white paper of the ACR incidental findings committee. J Am Coll Radiol. 2010;7:754-773.

29. Lee CI, Tsai EB, Sigal BM, Plevritis SK, Garber AM, Rubin GD. Incidental extracardiac findings at coronary $\mathrm{CT}$ : clinical and economic impact. AJR. 2010;194:1531-1538.

30. Wiener RS, Gould MK, Woloshin S, Schwartz LM, Clark JA. What do you mean, a spot? A qualitative analysis of patients' reactions to discussions with their physicians about pulmonary nodules. Chest. 2013;143:672-677.

31. Marshall HM, Bowman RV, Yang IA, Fong KM, Berg CD. Screening for lung cancer with low-dose computed tomography: a review of current status. J Thorac Dis. 2013;5(suppl):S524-S539.

32. Field JK, Oudkerk M, Pedersen JH, Duffy SW. Prospects for population screening and diagnosis of lung cancer. Lancet. 2013;382:732-741.

33. Heleno B, Thomsen MF, Rodrigues DS, Jorgensen KJ, Brodersen J. Quantifications of harms in cancer screening trials: literature review. BMJ. 2013;347:f5334.

34. Raftery J, Chorozoglou M. Possible net harms of breast cancer screening: updated modelling of Forrest report. BMJ. 2011;343:d7627.

35. Gil F, Costa G, Hilker I, Benito L. Quantification of harms in cancer screening trials: literature review. Stress Health. 2012;28:362-367.

36. MacHaalany J, Yam Y, Ruddy T, et al. Potential clinical and economic consequences of noncardiac incidental findings on cardiac computed tomography. J Am Coll Cardiol. 2009;54:1533-1541. 